\title{
PERFORMANCE EVALUATION OF VARIOUS IMAGE COMPRESSION TECHNIQUES USING SVD, DCT AND DWT
}

\author{
Anjali Ray ${ }^{1}$, Piyush Sharma ${ }^{2}$ \\ ${ }^{I}$ M. Tech scholar, Department of Electronics and Communication Engineering, S. S. College of Engineering, \\ Rajasthan, India \\ ${ }^{2}$ Assistant professor, Department of Electronics and Communication Engineering, S. S. College of Engineering, \\ Rajasthan, India
}

\begin{abstract}
Now a day, images are sending over the internet in very large amount. An uncompressed image requires more storage space and transmission bandwidth. So, the size of image database is increased. Image compression is a very active and mature field of research. Vast redundancy and the ability to absorb the moderate errors in the reconstruction of image make the compression possible. Considering the important role played by digital image and video, it is necessary to develop a system that produces high degree of compression while preserving critical image/video information. There is various transformation techniques used for data compression. Singular Value Decomposition (SVD), Discrete Cosine Transform (DCT) and Discrete Wavelet Transform (DWT) are the most commonly used Lossy compression techniques. In this work, we tested SVD, DWT, DCT algorithm for image compression comprehended using MATLAB code and modified to perform better when implemented in hardware description language.
\end{abstract}

Keywords: Image compression, SVD-DWT-DCT and MATLAB

\section{INTRODUCTION}

It is better said that "One picture is more powerful than 1000 of word". Image or picture is visual two or three dimensional recorded data information available either in raster or vector format. Now a day, with flourishing use of digitalization, digital image has been popular i.e. set of two dimensional array of pixel in numerical form. So in today's digitalized world every second millions of image are generated, recorded, decoded, stored and transmitted by various means[1]. Therefore, in jargon of flourishing data cyclone there is worthy need of reducing load of data trafficking for smother networking.

Image compression is basically process for reducing irrelevance and redundancy of the image data in order to be able to store or transmit data in an efficient form. It is achieved by using set of digitalized computational algorithm efficiently targeted to convert image size and color space without losing their image quality and other related information [2]. Basically, purpose of Image compression is to reduce the size of image and storage space and lower transmission time and bandwidth so that data cyclone can be reduced for better sailing in burgeoning ocean of modern data world.

\subsection{Principles of Image Compression}

Fundamentally camera use to record original object into either RGB or other color space in different format nevertheless image recorded in digital form represent array of pixel recorded in numerical form. Camera recorded image with color space is like naturally analog whilst digital image once converted and stored in pixel is like digital form. So, ultimately transition of analog to digital signal taking place that basically endowed with three types of possible data redundancy namely; Coding, Inter pixel (Spatial) and Psycho-visual redundancy [3].

Popularly image compression is mediated by two different methodology; Lossy and Lossless compression techniques [4]. In Lossless image compression, reconstructed image from compressed image is similar to original image. The noise cannot be added to signal or image. So, it is called noiseless. Lossy compression aims to reduce image size without any noise whilst lost certain image information nevertheless lossless targeted to no lose of data but affected by noise. Although Lossy compression lost redundant data technically, yet it's visually lossless and therefore has been more popularly implemented for routine image like JPEG and PNG [5][6].

It is necessary to preprocess digital uneven signal to square matrix form through numerical transformation and therefore image preprocessing is vital. Further, mostly image processing algorithm is targeted to spatial and psycho visual redundancy and function to overcome redundant data with either Entropy encoding (Lossless) or quantization (Lossy) process. As shown in Fig-1, basically first image is incorporated and color space conversion is implemented with RGB to $\mathrm{YCbCr}$ or other; it is carried out to remove unnecessary image density to reduce pixel distribution. However, it leads to noise conversion and may change transmission bandwidth. Later on using, image compression algorithm image is first partition into $\mathrm{n} \mathrm{X} \mathrm{n}$ block and each 
block is either process with encoding or quantization for further spatial redundancy [7]. Numerical matrix is further portioned into low pass to high pass filter across row and column masking for better image resolution and retention of image quality.

DCT, DWT and SVD are most popular and versatile image compression techniques used already in many study and reviewed in many previous work [8][9][10]. The approaches for Lossy compression include Lossy predictive coding and Transform coding. Fourier-related transform such as DCT and Wavelet Transform such as DWT are the most commonly used Lossy approach [11][12]. SVD is salient linear algebra based transformation that marks for superior quality maintenance of image following their compression. Power of SVD is their elimination of "zero value" and retention and reproduction of original image with "non zero" singular value square matrix. Technically in SVD vivid nature of image is code by each single value and geometry of image is sculptured by their vector function [13]. Discrete Wavelet Transform is a process of decomposition of image. This transformation is a function of small wave is called wavelet functions. DWT decompose image data in set of low pass and high pass coefficients. The Discrete Cosine Transform (DCT) has been applied extensively to the area of image compression. It has excellent energy-compaction properties and as a result has been chosen as the basis for the Joint Photography Experts Group (JPEG) still picture compression standard. DCT is an example of transform coding. The DCT coefficients are all real numbers and Inverse Discrete Cosine Transform (IDCT) can be used to retrieve the image from its transform representation. The DCT can be quickly calculated and is best for images with smooth edges. DCT is used to transform a signal from the spatial domain into the frequency domain [4]. DCT has the property that, for a typical image most of the visually significant information about an image is concentrated in just few coefficients of DCT. DCT is applied by some mathematical formulas. ZigZag scanning scans the DCT matrix in zig-zag manner. DCT takes less energy consumption [14].

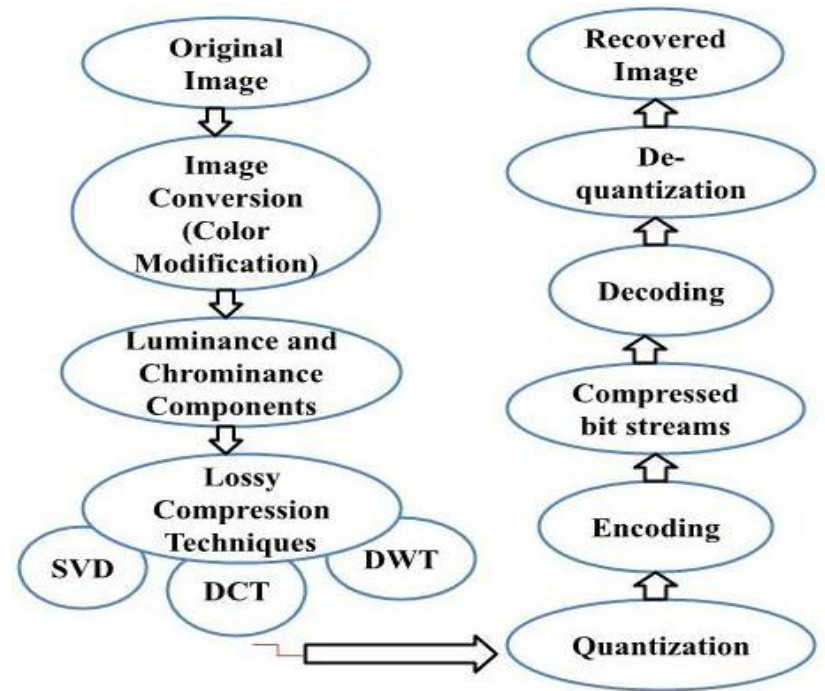

Fig-1: Block diagram of Image compression

\section{NEED OF WORK}

Multi faceted and objective oriented image transformation and compression with DCT, SVD and DWT is of the prime choice by many workers as discussed previously [15] [16][17]. Nevertheless many workers have sighted many pros and fame for listed compression method, yet very few works are reported where image with different object type like natural or artistic object based image analyzed simultaneously for testing consistency and supreme propounding of listed transformation method. Therefore, objective of our work is to test and compare power of each image compression method with different class of image so that it will be checked for their technical prophecy over wide image type available routinely and also have sighted pros and cons of each method based on our work.

We have studied and compare SVD, DCT and DWT transformation over different types of image with difference in their pixel size, object distribution and objective type whilst their performance were evaluated with statistically sound performance criteria as discuss further.

\subsection{Performance Criteria to be evaluated for Study}

As we have discussed earlier, image compression with lossy transformation causes partial loss of bits data signal for making process energy efficient for compression and lowering bits per symbol over all. Nevertheless bits data lost are there, we need to maintain quality of image without rate distortion for efficient image reconstruction. Therefore, it is vital to check performance and power of image compression algorithm using various performance indices and many parameters was previously consistently utilized and are as follow:

\subsubsection{Compression Ratio (CR): [2]}

The ratio between reconstructed image and original image size is called Compression Ratio. The value of compression ratio should be increased.

$$
\mathrm{CR}=\mathrm{n} 1 / \mathrm{n} 2 \ldots \ldots(1)
$$

Here, $\mathrm{n} 1$ is number of bits used to store original image and $\mathrm{n} 2$ is number of bits used to store compressed image.

Power of image compression is reflected with implicit compression ratio, better ratio indicates better compression, and hence always positive ratio is expected following study.

\subsubsection{Mean Square Error (MSE): [9]}

Mean Square Error is distortion rate in reconstructed image.

$$
\operatorname{MSE}=\frac{1}{M N} \sum_{i=1}^{M} \sum_{j=1}^{N}\left[X(i, j)-X^{\prime}\left(i_{i} j\right)\right] 2 \ldots \ldots
$$

Here, the $M, N$ is dimension of image. $X(i, j)$ is the pixel value of $(i, j)$ corresponding to original image and $X^{r}(\mathrm{i}, \mathrm{j})$ is the pixel value of reconstructed value. 
It is worthy to note that MSE is better estimate of disturbance and unevenness of system, hence more MSE indicate worsen image stability over algorithm transformation followed by their compression bits.

\subsubsection{Peak Signal - to - Noise Ratio [3]: [12]}

This is widely used quality measurement parameter. PSNR is most commonly used to measure the quality of reconstruction of lossy compression. A higher PSNR generally indicates that the reconstruction is of higher quality.

$$
\text { PSNR }=10 \log _{10} \frac{255}{M S E}(\mathrm{~dB}) \ldots \ldots(3)
$$

Here, 255 is the maximum possible value of pixel of image, here pixel is represented by 8 bit per sample so $2^{8}=256(0$ 255.)

\subsubsection{Elapsed Time (ET)}

What time is needed by algorithmic transformation following compression and reconstruction of image determine how much speedy algorithm is. Here, we have measured time for starting of execution of algorithm to their final completion in terms of seconds $(\mathrm{S})$ needed to complete work.

\subsection{Space Saving (\%): [17]}

Space saving (\%) determine performance of transformation efficiency over storage of data bits for original bit size to unprocessed bits size. It is similar to compression ratio however it reflects percentage of how much data space is saved following compression.

It is given by following equation:

$$
\mathrm{SS}(\%)=\left[1-\left(\frac{1}{\mathrm{CR}}\right)\right] * 100
$$

where $\mathrm{CR}=$ compression ratio

To estimate power of parameters further, all result were analyzed statistically using SPSS software for their tendency of central dispersion and other estimator.

\section{RESULT AND DISCUSSIONS:}

Image compression and their analysis in choosing energy efficient compression techniques is technically sound work carried out by many workers using different transformation method [18] [19]. In our work, experiment was carried out using two different types of natural and artistic class of image with each of two images in their respective category, as shown in data Table-1. Our image was choice with predetermination of two important things:

1. Color spectrum and color density of image

2. Distribution of main object and their repetitiveness in image.
Simply, image with single object is defined here as Natural image while scalar object distribution with repetitiveness of same object in image named as Artistic image class. Our aim was to test power of algorithm consistency of most popular DCT, DWT and SVD algorithm and their profound over wide background of image. Therefore, we have tested conventional algorithm using MATLAB coding for all four image.

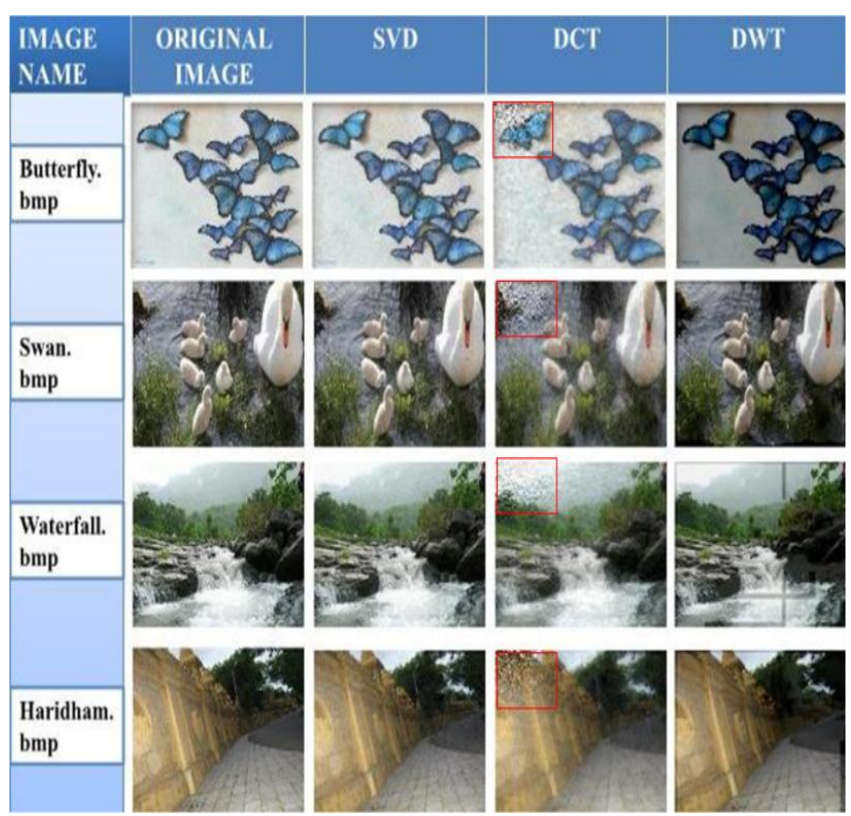

Fig-2: Depicting original bitmap image used in work and visual result of compressed image using SVD, DCT and DWT

Fig-2 is group of image depicting, original image of each work and image obtained following compression of each algorithm. From, visual analysis of image it become clear that in all case, SVD gives good compensate of image quality followed by DWT and DCT. One striking observation was that in all images, hue and saturation of color spectrum was almost visually intact in case of SVD while image was darker in DWT and in case of DCT slight blueness of vision is become apparent. Moreover, in DCT in All case, image compression artifact was observed in top left corner of image and image was shown to be slightly segmented in top marked corner (shown in red box). This problem may be due to improper image segmentation problem during inverse algorithm process. 


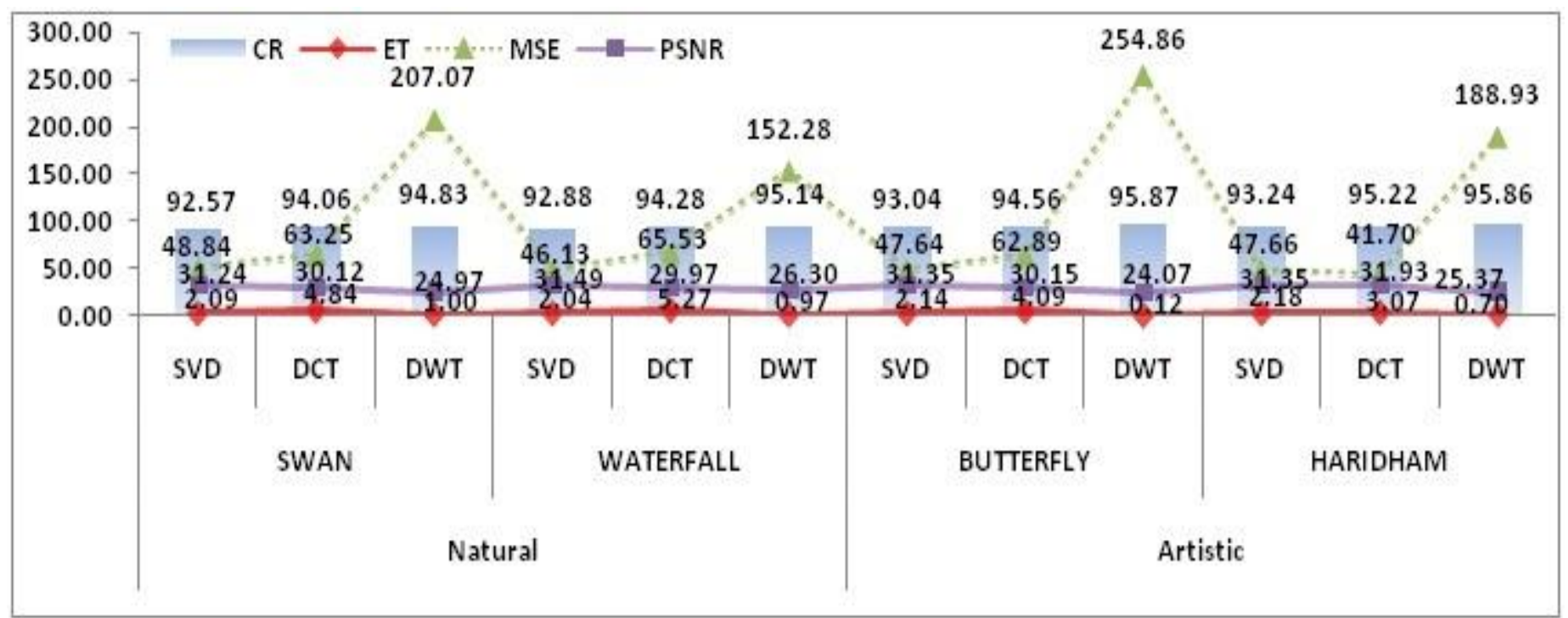

Graph 1: Graph depict relationship of image compression parameters over natural and artistic class image. Here, each class of image is marked with their type of algorithm used and figures in parenthesis indicate values of each parameter namely CR, ET, MSE and PSNR.

Table-1: Result of various parameters with different methods of compression techniques used

\begin{tabular}{|c|c|c|c|c|c|c|c|c|}
\hline Method & Parameter & SWAN & WATERFALL & BUTTERFLY & HARIDHAM & Mean & SD & CV $(\%)$ \\
\hline \multirow{5}{*}{ SVD } & SS (\%) & 98.92 & 98.92 & 98.93 & 98.93 & 98.92 & 0.00329 & 0.000033 \\
\hline & $\mathbf{C R}$ & 92.57 & 92.88 & 93.04 & 93.24 & 92.93 & 0.28 & 0.003053 \\
\hline & ET & 2.09 & 2.04 & 2.14 & 2.18 & 2.11 & 0.06 & 0.028583 \\
\hline & MSE & 48.84 & 46.13 & 47.64 & 47.66 & 47.56 & 1.11 & 0.023390 \\
\hline & PSNR & 31.24 & 31.49 & 31.35 & 31.35 & 31.36 & 0.10 & 0.003249 \\
\hline \multirow{5}{*}{ DCT } & SS (\%) & 98.94 & 98.94 & 98.94 & 98.95 & 98.94 & 0.01 & 0.000057 \\
\hline & CR & 94.06 & 94.28 & 94.56 & 95.22 & 94.53 & 0.50 & 0.005323 \\
\hline & ET & 4.84 & 5.27 & 4.09 & 3.07 & 4.32 & 0.96 & 0.223082 \\
\hline & MSE & 63.25 & 65.53 & 62.89 & 41.70 & 58.34 & 11.16 & 0.191273 \\
\hline & PSNR & 30.12 & 29.97 & 30.15 & 31.93 & 30.54 & 0.93 & 0.030444 \\
\hline \multirow{5}{*}{ DWT } & SS (\%) & 98.95 & 98.95 & 98.96 & 98.96 & 98.95 & 0.01 & 0.000058 \\
\hline & CR & 94.83 & 95.14 & 95.87 & 95.86 & 95.42 & 0.52 & 0.005497 \\
\hline & ET & 1.00 & 0.97 & 0.12 & 0.70 & 0.70 & 0.40 & 0.579679 \\
\hline & MSE & 207.07 & 152.28 & 254.86 & 188.93 & 200.78 & 42.65 & 0.212414 \\
\hline & PSNR & 24.97 & 26.30 & 24.07 & 25.37 & 25.18 & 0.93 & 0.036838 \\
\hline
\end{tabular}

Result of analysis is presented in Table-1 and Graph-1. From Graph-1 it becomes apparent that for all parameters, except MSE over all images, following every type of compression studied exhibits positive harmonic uniform trend. Moreover, our statistical analysis indicates that mean for PSNR lies in the range of $31.36 \pm 1.11$ (SVD) to $25.18 \pm 0.93$ (DWT) respectively. Among all the parameters; $\mathrm{CV}(\%)$ and Standard Deviation (SD) it is too much higher in case of parameter MSE especially in the DWT and overall in all case study indicates that the parameters MSE is highly influenced following an image compression and moreover it may be due to the problem in the algorithm compression and decompression process. More MSE indicates that Image stability is deteriorated following the compression [20]. 


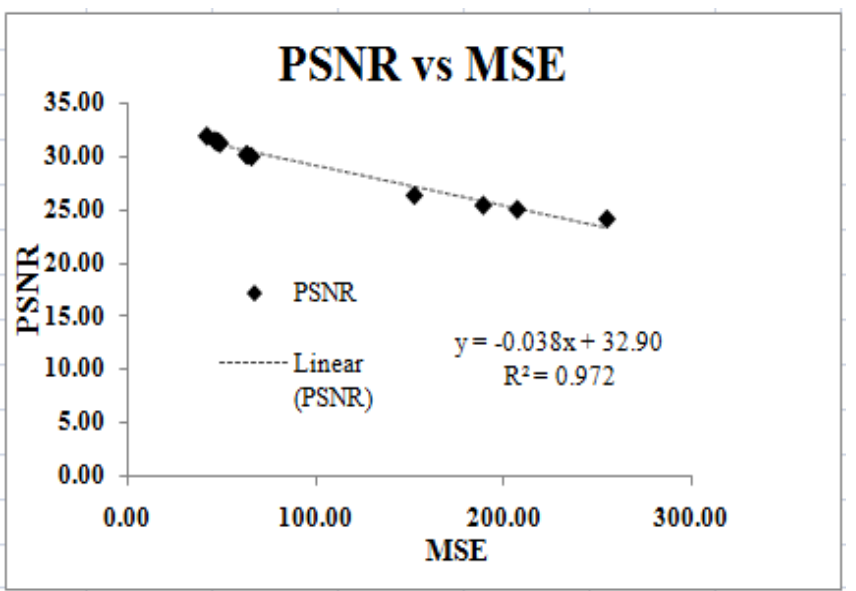

Graph 2: Graph depict relationship of PSNR Vs MSE with their trend and correlation for pooled data

Now days, storage capability and transmission bandwidth over work of image compression is major axioms of many works. Yet very few work in previous done have shown study related for it. We have cited importance to this objective, where parameter Space saving, in our study indicate consistent approximately $99 \%$ of space saved following compression work in all method tested.

From, above Graph-2 shows the relation between PSNR and MSE and it becomes distinct that whilst MSE approaching ascending manner towards the higher value, gradually PSNR is falling drop wise for all case. Moreover, it is clear also from equation of MSE that MSE is having inverse relationship with PSNR and our study is also in harmonic trend where perfect negative correlation is obtained with equation $\mathrm{Y}=-0.038 \mathrm{x}+32.9 \& \mathrm{R}^{2}=0.972 ;$ Also it is clearly reflected in Graph-2 that for the higher PSNR value; MSE is always low.

\section{CONCLUSION}

Image compression is highly useful and versatile technique which is popularly used now a day in the various formats using the methodology. From our analysis, we have tested the main three popular Lossy compression techniques and with two different class of image, it indicates that saliently PSNR and MSE are consistent and robust criteria for parameter studied. Space saving (\%) indicate that moreover $98 \%$ data storage space is saved which is highly appreciable. Although many workers has cited the algorithm works consistently, however we have shown, there is a certain issues in DWT i.e. it always reflect apex MSE for any type of image with partial yet noticeable image compression artifact as observed with DCT.

\section{REFERENCES}

[1]. K. C. Chandra Sekaran and Dr. K. Kuppusamy, Performance Analysis of Compression Techniques Using SVD, BTC, DCT and GP, IOSR Journal of Computer Engineering (IOSR-JCE) e-ISSN: 2278-0661, p-ISSN: 2278-8727, vol-17, Issue 1, ver. 2, Jan - Feb. 2015.
[2]. Archana Deshlahra, G. S. Shirnewar, and Dr. A. K. Sahoo, A Comparative Study of DCT, DWT \& Hybrid (DCT-DWT) Transform, 1st International Conference on Emerging Trends in Computer and Image Processing, June 2011.[https://www.researchgate.net/publication/282858254_ Comparative_study_of_DCT_DWT_and_Hybrid_technique s_for_Image_Compression].

[3]. Arshdeep Singh, Kamal Goyal, Navdeep Goel, and Vaibhav Phutela, Comparative Analysis of Image Compression Techniques using SVD, DCT and DWT, International Journal of Computer Applications, In International Conference on Communication, Computing and Virtualization (0975 - 8887), pp. 16-18, 2016.

[4]. Gonzalez and Woods, Digital Image Processing, 3rd edition, Dorling Kindersley (India) Pvt. Ltd, 2014.

[5]. A. M. Raid, W. M. Khedr, M. A. El-dosuky, and W. Ahmed, Jpeg Image Compression Using Discrete Cosine Transform-A Survey, arXiv preprint arXiv:1405.6147, 2014. [6]. Maini R., and Mehra S., A Review on JPEG2000 Image Compression. Int. J. Comput. Appl, vol-9, pp. 43-47, 2010.

[7]. Sebastian S., and Manimekalai M. A. P., Color image compression Using JPEG2000 with adaptive color space transform, In Electronics and Communication Systems (ICECS), International Conference on IEEE, pp. 1-5, 2014.

[8]. Subudhiray S., and Srivastav A. K., Implementation Of Hybrid Dwt-Dct Algorithm For Image Compression: A Review, 2012.

[9]. Barbhuiya A. H. M., Laskar T. A., and Hemachandran K., An Approach for Color Image Compression of JPEG and PNG Images Using DCT and DWT, In Computational Intelligence and Communication Networks (CICN), 2014 International Conference on IEEE, pp. 129-133, 2014.

[10]. Jayaraman S, Veerkumar T, Esakkirajan S, Digital Image Processing, Tata McGraw Hill Education, 2009.

[11]. B. Chanda, and Dutta Majumder, Digital Image Processing and Analysis, PHI Learning Private Limited, Delhi, 2011.

[12]. Raghavendra .M. J, Prasantha .H. S, and Sandya S, DCT SVD Based Hybrid Transform Coding for Image Compression, International Journal on Recent and Innovation Trends in Computing and Communication, vol3, no.-6, pp. 4066-4071, 2015.

[13]. V. C. Klema, The singular value decomposition: its computation and some applications, IEEE Transactions in automatic control, vol-25, pp. 164-176, 1980.

[14]. Delp E.J, and Mitchell O.R., Image Compression using Block Truncation Coding, IEEE Trans. Communications, vol-27, pp. 1335-1342, 1979.

[15]. Fouzi Douak, Color Image Compression Algorithm Based On The DCT Transform Combined To An Adaptive Block Scanning, IEEE Transactions On Information Forensics And Security, vol-6, no.-3, 2011.

[16]. Ahmed N., Natarajan T., and Rao, K. R., Discrete Cosine Transform, IEEE Transactions on Computers, vol100, no.-1, pp. 90-93, 1974.

[17]. https://en.wikipedia.org/wiki/Data_compression_ratio, as accessed on 18 May, 2017 at 11:30 AM.

[18]. Charles F. Hall, A Hybrid Image Compression Technique, CH2I 18-8/85/0000-0149, IEEE, 1985. 
[19]. P. Hong, and S. W. Bao, Hybrid image compression model based on subband coding and edge preserving regularization, Vision, Image and Signal Processing, IEEE Proceedings, vol-147, Issue: 1, pp. 16-22, 2000.

[20]. W. Khan, Image Segmentation Techniques: A Survey, Journal of Image and Graphics, vol-1, no.-4, pp. 166-170, 2013. 